\title{
Brilliant Green Staining of the Fingernails
}

\author{
Khatiya Chelidze, MD; Shari R. Lipner, MD, PhD
}

\section{PRACTICE POINTS}

- Chloronychia, or green nail syndrome, is due to Pseudomonas aeruginosa infection and is a common etiology of green nail discoloration. Green nail discoloration also may be secondary to use of the antiseptic dye brilliant green.

- Brilliant green is bactericidal but has no known antifungal or anti-inflammatory activity; it should be considered in the differential diagnosis of green nail discoloration and also may cause blindness with eye contact.

We present a case of brilliant green staining of the fingernails caused by the patient's use of an antiseptic dye to self-treat onychodystrophy. Brilliant green is an antiseptic dye used since at least the early 20th century for the treatment of bacterial infections. It was originally used for wound care and as an anti-inflammatory medication; however, its use for both of these indications is limited. Because of its ease of use, low cost, and accessibility, brilliant green has been promoted for use in resource-poor settings and as an anticancer therapy. Dermatologists should be aware of brilliant green, as well as its indications, common uses, and adverse effects, and should be able to differentiate it from other causes of green nails.

Cutis. 2020;105:317-318.

\section{Case Report}

A 92-year-old Eastern European woman presented to our nail clinic with a history of onychodystrophy and arthralgia of the digits of several months' duration. Her dermatologic history was notable for irritant hand dermatitis. A prior nail plate clipping with histopathologic examination was negative for fungal elements. Physical examination revealed onychorrhexis of all fingernails as well as onycholysis and subungual hyperkeratosis of the right fourth fingernail. Blue-green staining was incidentally noted on the right second and third fingernails and nail folds (Figure 1). Contact dermoscopy using ultrasound gel revealed translucent areas with sparse pigment, though denser areas had a fine branching pattern (Figure 2). When questioned, the patient reported use of "zelyonka," a brilliant green solution, to selftreat the nails. Histopathology on repeat nail clippings showed parakeratosis and serum, which was most consistent with her known history of irritant hand dermatitis. Radiographs of the hands revealed osteoarthritis that was most prominent at the distal interphalangeal joints.

\section{Comment}

Brilliant green is a triphenylmethane dye commonly used in Eastern Europe and other regions for the treatment of superficial skin infections and onychomycosis. ${ }^{1}$ Its use as an antiseptic and wound healing agent has been investigated in the scientific literature since at least the early 20 th century. ${ }^{2}$ Brilliant green typically is applied in a $0.1 \%$ to $2 \%$ ethanol solution. ${ }^{1}$ The dye has bactericidal

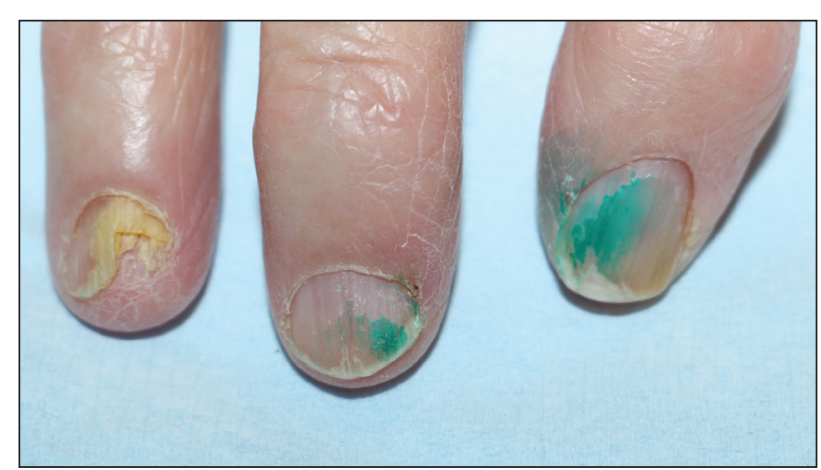

FIGURE 1. Blue-green staining on the right second and third fingernails and nail folds from use of brilliant green to self-treat onychodystrophy. 


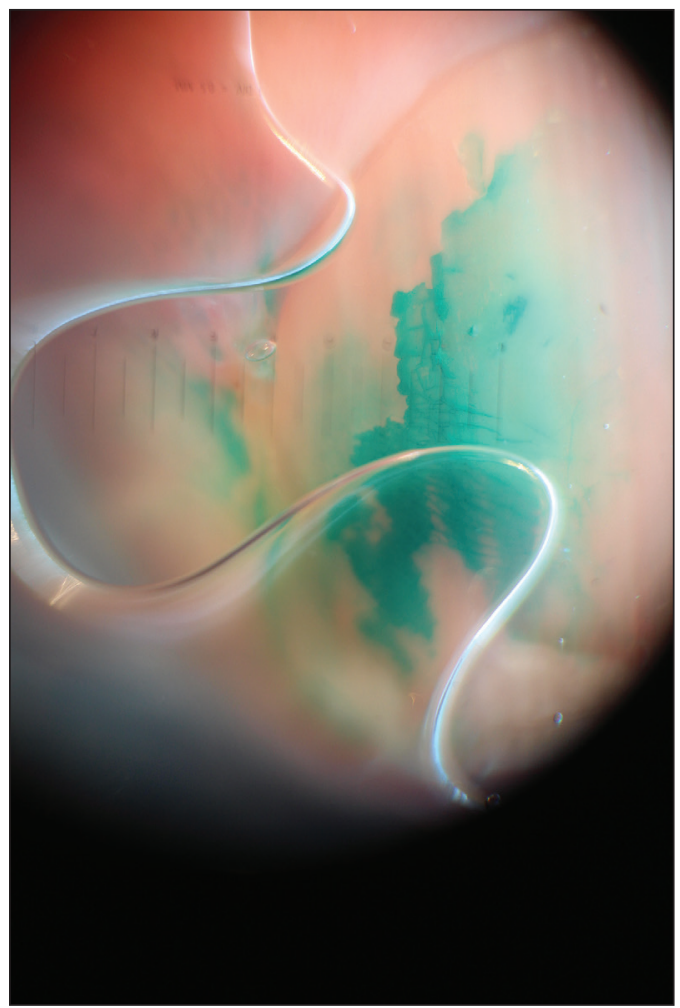

FIGURE 2. Contact dermoscopy showed translucent areas with sparse pigment and denser areas with a fine branching pattern.

activity against gram-positive organisms, particularly staphylococci and streptococci. ${ }^{2,3}$ It has been used for the treatment of fungal skin and nail infections since at least the early 20th century, with anecdotal success. ${ }^{4}$ Although there have been no studies investigating use of brilliant green alone for the treatment of onychomycosis, it is sometimes used in combination with conventional oral agents for this purpose. ${ }^{5}$ Because of its availability, safety, ease of use, and low cost, brilliant green has been promoted as an antiseptic in resource-poor settings. ${ }^{3}$ The revival of brilliant green and other antiseptic dyes in these settings has been suggested as an alternative to oral antibiotic agents, to which resistance is rising, and as a potential cancer therapy. ${ }^{6,7}$ Although brilliant green's mechanism of action in treating skin infections is unclear, it has been shown to form covalent adducts with thioredoxin reductase 2, a protein conserved from bacteria to humans with an essential function for cellular activity. ${ }^{7}$
Early case studies suggested that brilliant green was beneficial in treating wounds ${ }^{2}$; however, this indication is controversial. In a guinea pig study, brilliant green was shown to inhibit wound healing and the formation of granulation tissue. ${ }^{8}$ It also should be noted that when used topically, brilliant green may cause skin sensitization, necrotic skin reactions, and permanent staining of clothing. It has no known anti-inflammatory properties and also may cause skin irritation. ${ }^{8}$ Brilliant green may cause blindness if it comes in contact with the eyes. ${ }^{1}$

Brilliant green has other potential dermatologic indications. For example, a combination of brilliant green and gentian violet, a related dye, has demonstrated efficacy in the treatment of cutaneous hemangiomas in mouse models by blocking expression of angiopoietin-2. ${ }^{7}$

Dermatologists should be familiar with brilliant green and its common uses as well as adverse effects. Brilliant green is commercially available for a low cost (\$5 to \$20) in specialty pharmacies or online (eg, Amazon). It is sold alone or in combination with gentian violet and proflavine hemisulfate, and a prescription is not required. Due to its low cost and accessibility, patients may use brilliant green to self-treat dermatologic conditions. Green nails due to staining with brilliant green dye must be distinguished from other etiologies causing green nail discoloration, such as infection with Pseudomonas aeruginosa or Aspergillus, bullous disorders, jaundice, "old" hematomas, nail polish, and other exogenous pigments.

\section{REFERENCES}

1. Balabanova M, Popova L, Tchipeva R. Dyes in dermatology. Clin Dermatol. 2003;21:2-6.

2. Browning $\mathrm{CH}$, Gulbransen $\mathrm{R}$, Kennaway EL, et al. Flavine and brilliant green, powerful antiseptics with low toxicity to the tissues: their use in the treatment of infected wounds. Br Med J. 1917;1:73-78.

3. Bakker P, Doorne H, Gooskens V, et al. Activity of gentian violet and brilliant green against some microorganisms associated with skin infections. Int J Dermatol. 1992;31:210-213.

4. Montgomery RM, Casper EA. Cutaneous manifestations of the fungi causing dermatophytosis and onychomycosis and their treatment. J Am Med Assoc. 1945;128:77-83.

5. Tchernev G, Cardoso JC, Ali MM, et al. Primary onychomycosis with granulomatous Tinea faciei. Braz J Infect Dis. 2010;14:546-547.

6. Berrios RL, Arbiser JL. Effectiveness of gentian violet and similar products commonly used to treat pyodermas. Dermatol Clin. 2011; 29:69-73.

7. Maley AM, Arbiser JL. Gentian violet: a 19th century drug re-emerges in the 21st century. Exp Dermatol. 2013;22:775-80.

8. Niedner R, Schöpf E. Inhibition of wound healing by antiseptics. Br J Dermatol. 1986;115:41-44 